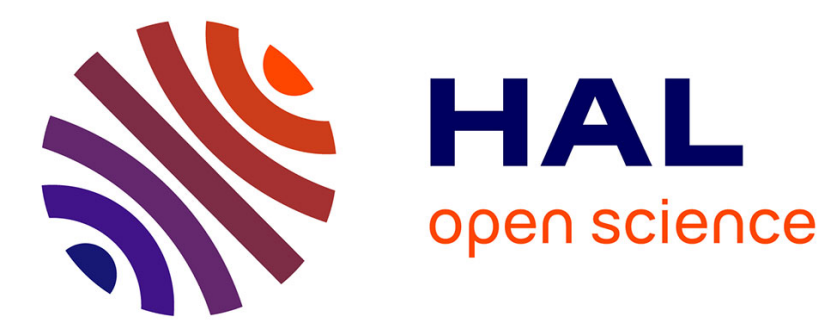

\title{
Croissance et structure des couches minces
}

\author{
A. Marty, S. Andrieu
}

\section{To cite this version:}

A. Marty, S. Andrieu. Croissance et structure des couches minces. Journal de Physique IV Proceedings, 1996, 06 (C7), pp.C7-3-C7-11. 10.1051/jp4:1996701 . jpa-00254489

\section{HAL Id: jpa-00254489 https://hal.science/jpa-00254489}

Submitted on 1 Jan 1996

HAL is a multi-disciplinary open access archive for the deposit and dissemination of scientific research documents, whether they are published or not. The documents may come from teaching and research institutions in France or abroad, or from public or private research centers.
L'archive ouverte pluridisciplinaire HAL, est destinée au dépôt et à la diffusion de documents scientifiques de niveau recherche, publiés ou non, émanant des établissements d'enseignement et de recherche français ou étrangers, des laboratoires publics ou privés. 
JOURNAL DE PHYSIQUE IV

Colloque C7, supplément au Journal de Physique III, Volume 6, novembre 1996

\title{
Croissance et structure des couches minces
}

\author{
A. Marty et S. Andrieu* \\ CENG, Département de Recherche Fondamentale sur la Matière Condensée, SPMM/NM, 38054 Grenoble \\ cedex 09, France \\ * Laboratoire de Physique du Solide, Université H. Poincaré Nancy 1, BP. 239, \\ 54506 Vandouvre-les-Nancy cedex, France
}

\begin{abstract}
Résumé : Nous présentons dans ce papier sans prétendre être exhaustifs tant le thème est vaste, quelques techniques d'obtention des couches minces les plus utilisées dans les laboratoires de recherche, les grands types et processus de croissance notamment pour les couches minces obtenues par évaporation sous vide, l'épitaxie et quelques mécanismes de relaxation des contraintes.
\end{abstract}

\section{INTRODUCTION}

L'étude des propriétés magnétiques de films minces métalliques (quelques $\AA$ à $1000 \AA$ d'épaisseur) est une activité récente puisqu'elle a réellement démarré il y a un peu plus d'une dizaine d'années. Son essor a toutefois été considérable, en particulier parce que les techniques de fabrication ont permis d'atteindre des degrés de précision fantastique dans le contrôle des épaisseurs déposées. Contrôler l'épaisseur d'une couche de $10 \AA ̊$ à $1 \AA$ près constitue actuellement une opération tout à fait banale pour les spécialistes de ces techniques de dépôt. Ainsi, il est devenu rapidement possible de construire des objets magnétiques de tailles très réduites comme des sandwiches "métal magnétique / métal non-magnétique / métal magnétique" afin d'observer le couplage entre les deux couches magnétiques au travers de l'espaceur que constitue le métal non-magnétique de quelques Angströms d'épaisseur. Grâce à de tels objets, la magnétorésistance géante a été découverte en 1988 par une équipe française dans le système $\mathrm{FeCr}$ [1]. Cette découverte a constitué une petite révolution car ce mécanisme est maintenant utilisé dans la fabrication de têtes magnétorésistives très sensibles pour la lecture de bandes magnétiques. D'autre part, grâce à l'énergie magnétique d'interface entre deux matériaux déposés l'un sur l'autre, il est possible d'obtenir une anisotropie magnétique perpendiculaire au plan des couches. Cette anisotropie permet ainsi de réduire la taille des domaines et d'envisager un stockage plus dense de l'information. Enfin, il est devenu possible, grâce aux techniques d'évaporation sous ultravide, de réaliser des films minces épitaxiés sur un support monocristallin, ce qui a ouvert la voie à l'étude des propriétés magnétiques de matériaux présentant des structures cristallographiques nouvelles dues entre autres aux contraintes de cohérence imposées par le substrat. En particulier, l'étude de la variation des propriétés magnétiques des métaux magnétiques $3 \mathrm{~d}$, de terres rares, ou bien de matériaux non magnétiques comme les $4 \mathrm{~d}$ et les $5 \mathrm{~d}$, est devenue possible.

On peut ainsi distinguer plusieurs thèmes d'études, a priori différents, comme : le couplage magnétique (épaisseur des couches de quelques Angströms, interaction de type RKKY), intéressant pour l'étude de la magnetorésistance géante ou/et pour l'étude de dispositif à base de métaux magnétiques utilisant la variation de la résistivité de la couche magnétique (résistance variable, mémoire, dispositif à injection de spins polarisés, etc....) ; l'anisotropie magnétique pour l'étude de couches minces à aimantation perpendiculaire ; les relations entre le magnétisme, la structure cristallographique et les contraintes élastiques, afin de comprendre les comportements magnétiques de matériaux synthétisés dans des structures cristallographiques que l'on ne rencontre pas dans la nature; ou encore les nanostructures où les objets magnétiques ont des dimensions réduites dans les trois directions de l'espace. Dans la pratique, la fabrication des matériaux dont les propriétés magnétiques spécifiques dépendent des conditions de croissance, de la structure, de la morphologie des couches, permet l'étude de tous ces thèmes. De plus, il existe un objectif commun à tous ces thèmes : on a souvent besoin d'obtenir des dépôts avec des interfaces 
les plus planes possibles. Nous insisterons donc naturellement dans cet article sur les différents mécanismes conduisant à une rugosité d'interface.

Différentes techniques de synthèse sont utilisées selon les thèmes d'étude. Bien souvent, il n'est pas nécessaire de fabriquer des films minces monocristallins, donc épitaxiés. Il est donc utile dans un premier temps de passer en revue les principales techniques de synthèse de films minces métalliques utilisées actuellement. En fait, il est rapidement apparu que les techniques a priori les plus "simples" à mettre en oeuvre comme la pulvérisation cathodique ou l'évaporation sous vide moyen par exemple, ne permettent pas encore actuellement de fabriquer des systèmes modèles simples, utiles pour la compréhension des phénomènes physiques. Les dépôts étant polycristallins, il est ainsi particulièrement difficile d'étudier les mécanismes de croissance des métaux en films minces fabriqués par ces techniques. Au contraire, les techniques d'épitaxie sous ultravide sont souvent bien adaptées à ces études car d'une part il est possible de travailler sur des systèmes modèles et, d'autre part, un nombre important de techniques d'analyse fines insitu sont disponibles sur ces appareillages. Pour mieux comprendre, citons un exemple montrant l'intérêt de travailler avec des systèmes modèles : des contradictions "apparentes" ont été relevées dans la littérature au sujet des périodes d'oscillation du couplage anti-ferromagnétique entre deux couches magnétiques séparées par un "espaceur". Bruno et Chappert ont alors montré que la période de ces oscillations dépend de la direction cristallographique selon laquelle se propagent les électrons [2]. Ainsi, pour une couche texturée (100) ou (111), la période n'est pas la même. S'il est difficile de contrôler la texture d'un dépôt polycristallin en pulvérisation cathodique, il n'en est pas de même en épitaxie, et la vérification des prédictions théoriques a pu être menée sans ambiguïté. Ainsi, notre savoir sur les mécanismes de croissance des métaux en film minces provient essentiellement d'études réalisées sur des dépôts épitaxiés, bien que la pulvérisation cathodique ou l'évaporation sous vide moyen soient beaucoup plus utilisés parce que la qualité des systèmes fabriqués est souvent suffisante.

\section{TECHNIQUES DE DÉPÔT}

Les méthodes d'élaboration de couches minces sont très variées. Sans prétendre faire une classification exhaustive, on peut distinguer les méthodes physiques, telles que l'évaporation ou la pulvérisation, des méthodes chimiques, en phase vapeur ou liquide. Les méthodes physiques, notamment l'évaporation sous ultra vide, sont essentiellement utilisées par les laboratoires de recherche car elles permettent d'élaborer des matériaux très divers et de mesurer in-situ les paramètres physiques. Les méthodes chimiques, plus spécialisées, sont par contre beaucoup plus intéressantes pour les fabrications en série de composants industriels quand cela est possible.

\subsection{Méthodes chimiques.}

\subsubsection{Croissance en phase liquide}

L'électrodéposition et/ou anodisation, déjà largement utilisées dans l'industrie (par exemple pour le revêtement), sont maintenant aussi utilisées pour l'élaboration de multicouches métalliques. Elles nécessitent l'utilisation d'un électrolyte et de séquences de polarisation alternative des électrodes adaptées à chaque type de matériaux. De gros progrès ont été obtenus récemment pour la réalisation de multicouches, l'intérêt de cette technique étant sa simplicité de mise en oeuvre par rapport aux techniques lourdes usuelles.

\subsubsection{Croissance en phase vapeur}

Outre la croissance thermique ou l'oxydation, la CVD (Chemical Vapor Déposition) est largement répandue. Comme pour l'électrodéposition pour laquelle le choix de l'électrolyte est crucial, elle nécessite un vecteur de transport adapté à chaque matériau à déposer. Par exemple on peut obtenir un dépôt de nickel ou de platine à partir des réactions :

$$
\begin{gathered}
\mathrm{Ni}(\mathrm{CO})_{4}--->\mathrm{Ni}+4 \mathrm{CO}\left(\text { de } 100 \text { à } 300^{\circ} \mathrm{C}\right) \\
\mathrm{Pt}(\mathrm{CO})_{2} \mathrm{Cl}_{2}--->\mathrm{Pt}+2 \mathrm{CO}+\mathrm{Cl}_{2}\left(250^{\circ} \mathrm{C}\right)
\end{gathered}
$$

L'étude de l'homogénéité et des caractéristiques des dépôts dans un réacteur CVD est complexe et conduit à une modélisation numérique couplant la mécanique des fluides, la thermique et la cinétique de réaction chimique. Les méthodes chimiques permettent d'obtenir un dépôt sur toutes les faces d'un objet ce qui 
présente un intérêt pour certaines applications, contrairement aux méthodes physiques d'évaporation ou de pulvérisation qui sont plus directionnelles.

\subsection{Méthodes Physiques}

\subsubsection{L'évaporation ou sublimation sous vide moléculaire}

On parle de vide moléculaire quand les atomes ou les molécules du flux d'évaporation ont une probabilité de rencontre faible avant d'atteindre le substrat, $c^{\prime}$ est à dire quand le libre parcours moyen est très supérieur aux dimensions de la chambre de dépôt. A titre d'exemple, à $25^{\circ} \mathrm{C}$, le libre parcourt moyen est de l'ordre de $5 \mathrm{~m}$ à $10^{-5}$ Torr et de $50 \mathrm{~km}$ à $10^{-9}$ Torr !. Dans le vide moléculaire, les flux sont directionnels, et les espèces chimiques n'interagissent que sur le substrat. L'ultravide (pression $<10^{-9}$ Torr) permet aussi de limiter l'incorporation d'impuretés lors du dépôt ; à 10-9 Torr, il faudrait 40 minutes au minimum pour remplir une couche atomique avec les espèces résiduelles (et indésirables) présentes dans la chambre. A une pression de $10^{-5}$ Torr, ce temps est ramené à $1 / 5$ seconde !.

On peut différencier les techniques d'évaporation par le type d'évaporateur utilisé :

1.2.1.1 fil résistif : un fil de tungstène ou de tantale enrobé d'un métal plus volatil est porté à haute température par le passage d'un courant électrique. Facile à mettre en oeuvre pour un coût et un encombrement réduit, cette technique est limitée aux faibles épaisseurs déposées car le fil est rapidement consommé. Elle est utilisée pour l'étude des premiers stades de croissance.

1.2.1.2 Cellule de Knudsen : le métal à évaporer est placé dans un creuset suffisamment réfractaire porté à la température d'évaporation par chauffage inductif ou résistif. Si le trou d'effusion est suffisamment petit, l'intérieur de la cellule est à l'équilibre thermodynamique. Il règne alors une pression uniforme $\mathrm{P}$ que l'on peut calculer si on connaît l'activité de la phase condensée, acond, et l'enthalpie libre de vaporisation ( $\Delta \mathrm{G})$.

$$
\frac{P}{a_{\text {cond }}}=e^{-\frac{\Delta G(\text { cond }->\text { gas })}{k T}}
$$

On peut alors calculer les flux moléculaires sortant du trou d'effusion à partir de la formule de Hertz-knudsen [3], pour une espèce chimique de masse atomique $\mathrm{M}$, dans la cellule portée à la température T et à la pression P:

$$
\text { Flux }=\frac{\mathrm{P}}{\sqrt{2 \pi \mathrm{MkT}}}=3.510^{22} \frac{\mathrm{P}}{\sqrt{\mathrm{MT}}} \text { molécules. } \mathrm{cm}^{-2} \mathrm{~s}^{-1}
$$

Le flux de la cellule de Knudsen est stable si la température est asservie.

1.2.1.3 L'évaporateur à bombardement électronique : une charge du métal à évaporer est placée dans un creuset en cuivre généralement refroidi par une circulation d'eau. La charge est soumise à un bombardement électronique localisé sur sa surface. La température de la surface est très inhomogène et peu stable ; les flux peuvent donc fluctuer. Pour réaliser des dépôts précis, ce type d'évaporateur doit être régulé par une mesure continue des flux émis, par exemple en utilisant une balance à quartz. Contrairement aux cellules de Knudsen limitées pour les éléments les plus réfractaires, les évaporateurs à bombardement électronique permettent d'évaporer les éléments les plus réfractaires. Cependant, comme pour les cellules de Knudsen, si l'on cherche à évaporer des composés (alliages), il n'est pas rare de se trouver confronté au phénomène d'évaporation préférentielle, qui conduit à une variation de la composition de la charge, et donc des flux, en cours d'utilisation prolongée.

1.2.1.4 L'ablation laser : la surface de la charge est irradiée par un faisceau laser pulsé, la densité d'énergie absorbée est très grande et le système est largement hors d'équilibre. Cette méthode peut aussi fonctionner avec des composés et les flux émis conservent la stoechiométrie de la charge. Cependant, le processus est tellement violent que le flux peut contenir des agrégats ou des gouttes.

\subsubsection{La pulvérisation cathodique}

Le principe de base de la pulvérisation cathodique est assez simple (figure 1) : c'est une diode formée par la charge (cible) à pulvériser (cathode) et le substrat (anode), placée dans une enceinte contenant un gaz neutre tel que l'argon. Une tension de quelques $\mathrm{kV}$ appliquée entre l'anode et la cathode entraîne une décharge auto-entretenue si la pression de gaz neutre est appropriée (10 à $500 \mathrm{mT}$ Torr). Les ions du gaz neutre 
bombardent violemment la cible polarisée négativement, pulvérisant ainsi les atomes de surface qui vont se déposer sur le substrat. A ce dispositif de base on peut adjoindre des effets additionnels qui permettent d'en augmenter l'efficacité :

1.2.2.1 Le procédé triode : une source auxiliaire d'électrons (thermo-émission) correctement polarisée permet d'entretenir le plasma pour une pression de gaz rare plus faible (0.1 mTorr).

1.2.2.2 Le procédé magnétron : Un confinement magnétique du plasma près de la cible, par exemple avec des aimants permanents correctement disposés, permet d'augmenter nettement le rendement d'ionisation des électrons qui effectuent des trajectoires hélicoïdales. $\mathrm{Ce}$ dispositif permet aussi de baisser la pression tout en

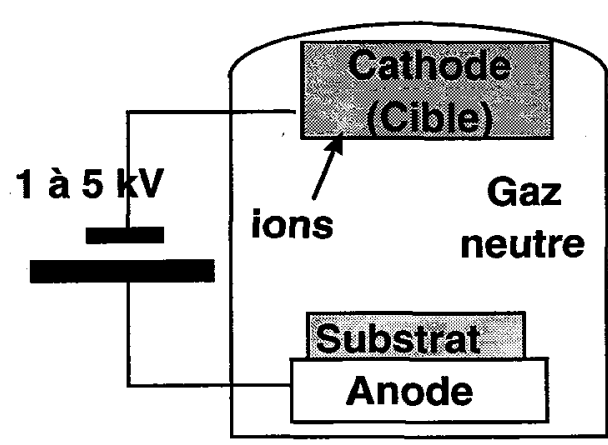

figure 1: Schéma de principe d'un dispositif de pulvérisation cathodique (procédé điode) maintenant la décharge $(0.5$ à 5 mTorr).

1.2.2.3 La radio fréquence : le procédé de base ne permet pas la pulvérisation de cible faite d'un matériau isolant électrique; elle se polarise et repousse les ions qui ne peuvent plus atteindre sa surface. Pour pallier cet effet, il faut appliquer une tension radiofréquence (13 ou $27 \mathrm{MHz}$ ) pour la dépolariser.

L'intétêt de la pulvérisation cathodique réside dans sa simplicité et sa robustesse, mais aussi dans sa capacité à pulvériser des composés sans altérer la stoechiométrie.

\section{LES MÉCANISMES DE CROISSANCE}

\subsection{Modes de croissance}

Classiquement, on distingue 3 modes de croissance :

\subsubsection{La croissance bidimensionnelle (dite de Frank Van-der-Merve)}

Sur une face d'un cristal, les couches atomiques croissent les unes après les autres et la surface reste atomiquement plane au cours du dépôt. En terme d'énergie superficielle, on s'attend à avoir ce type de croissance si : $\gamma_{\mathrm{sv}}>\gamma_{\mathrm{Sd}}+\gamma_{\mathrm{vd}}$, où $\mathrm{s}$, v et $\mathrm{d}$ désignent respectivement le substrat, le vide et le dépôt.

\subsubsection{La croissance tridimensionnelle (dite de Volmer-Weber)}

Cette fois-ci, il y a formation d'îlots formés de plusieurs couches atomiques (figure 2). A l'équilibre thermodynamique, on peut relier les énergies superficielle et interfaciale à la forme de l'îlot à l'aide de la formule de Young (a) [4] ou du théorème de Wulff (b), selon l'anisotropie de l'énergie superficielle [5].

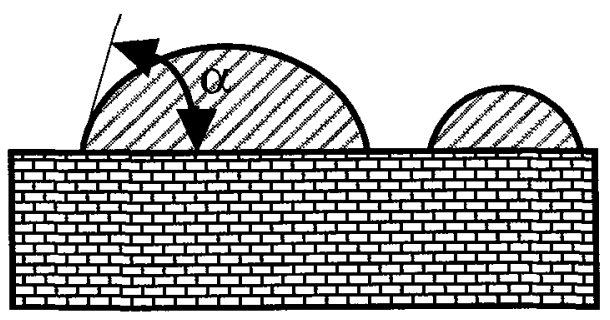

a) $\gamma_{\mathrm{sv}}=\gamma_{\mathrm{sd}}+\gamma_{\mathrm{vd}} * \operatorname{Cos}(\alpha)$

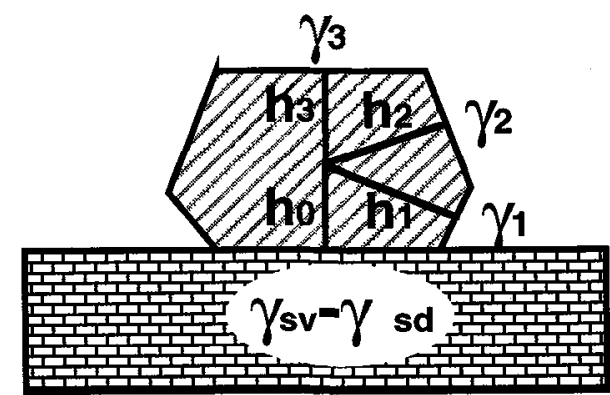

b) $\frac{\gamma_{\mathrm{sv}}-\gamma_{\mathrm{sd}}}{\mathrm{h}_{0}}=\frac{\gamma_{1}}{\mathrm{~h}_{1}}=\frac{\gamma_{2}}{\mathrm{~h}_{2}}=\frac{\gamma_{3}}{\mathrm{~h}_{3}}$

figure 2 : Relation entre la forme de l'îlot et les énergies superficielle et interfaciale dans la croissance tridimensionnelle 


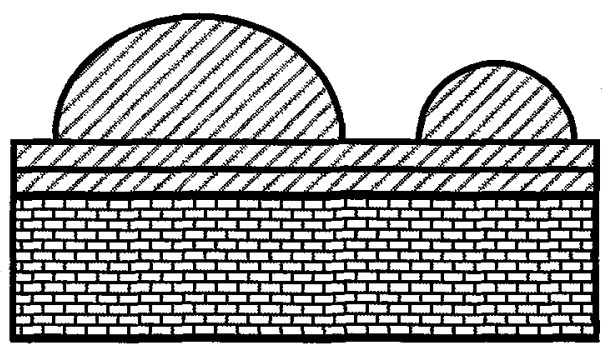

figure 3 : mode de croissance de Stranski-Krastanov

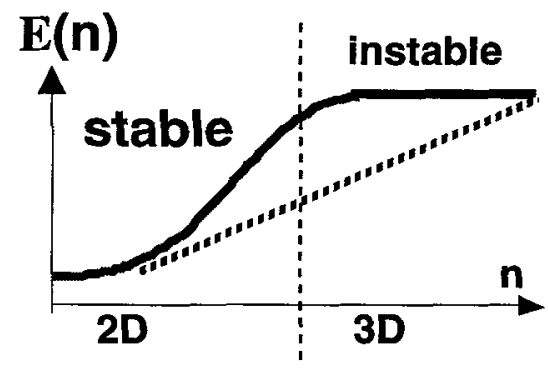

figure 4 : énergie en fonction du nombre de couche présentant un point d'inflexion

\subsubsection{La croissance de Stranski-Krastanov}

C'est une croissance qui débute comme celle de Frank Van-der-Merve (Croissance 2D) mais après quelques couches atomiques, la croissance devient tridimensionnelle (figure 3). On peut modéliser la transition de régime de croissance à partir de la courbe donnant l'énergie de la couche déposée en fonction du nombre de couches, $\mathrm{n}$ (figure 4). Si elle présente un point d'inflexion, le système sera instable vis à vis de fluctuations de hauteurs au-delà de ce point d'inflexion, ce qui tendra vers la formation d'îlots $3 \mathrm{D}$ quand l'épaisseur critique sera dépassée.

\subsection{Processus élémentaires}

Les descriptions précédentes sont basées sur des considérations d'équilibre thermodynamique. Mais pour réellement comprendre la croissance, il faut s'intéresser aux processus élémentaires au niveau atomique. La figure 5 en donne une idée schématique. Les espèces provenant du flux incident sont adsorbées, elles peuvent aussi être désorbées. La désorption étant thermodynamiquement activée est négligeable pour les métaux que l'on dépose à température ambiante ou pas trop élevée. Si les atomes restaient sur le site d'impact, la fluctuation du flux incident entraînerait une rugosité rapidement croissante. On pourrait modéliser cette croissance en colonnes atomiques (SOS : solid on solid) sans interaction soumises à un flux poissonnien d'atomes ; la rugosité serait l'écart-type de cette distribution, c'est à dire qu'elle serait proportionnelle à la racine carrée de l'épaisseur.

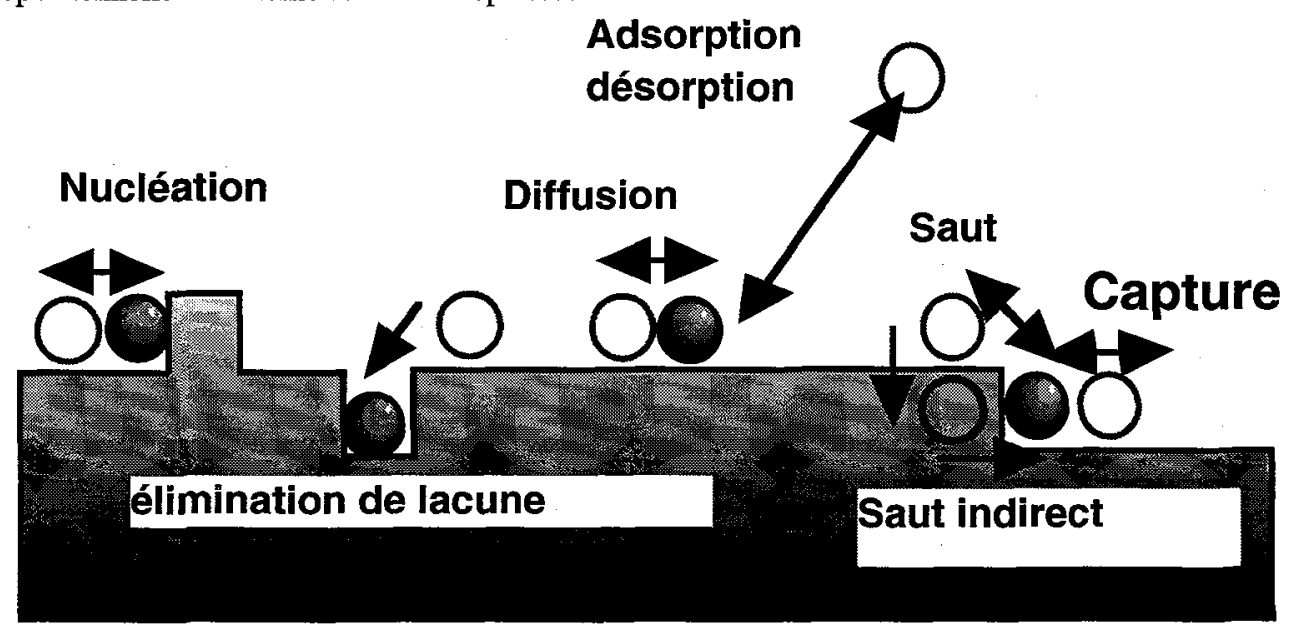

figure 5 : les processus élémentaires à l'oeuvre lors de la croissance

Mais la diffusion atomique sur la surface (ad-atomes) assure une redistribution des atomes. La diffusion des ad-atomes est bien plus facile que la diffusion en volume (énergie d'activation plus faible) et même à température ambiante, elle est active pour les métaux. Cependant, il existe des mécanismes de piégeage des 
ad-atomes qui vont limiter l'effet de lissage de la surface par la diffusion. Ces pièges sont liés à la nature discrète du réseau cristallin : adsorption d'un ad-atome dans une ad-lacune, nucléation d'îlots de taille suffisante pour être stables (dimères, trimères etc..), capture d'un ad-atome par une marche montante ou descendante, etc. Le processus de capture par une marche peut être très dissymétrique, car lors de la descente de la marche, l'ad-atome se

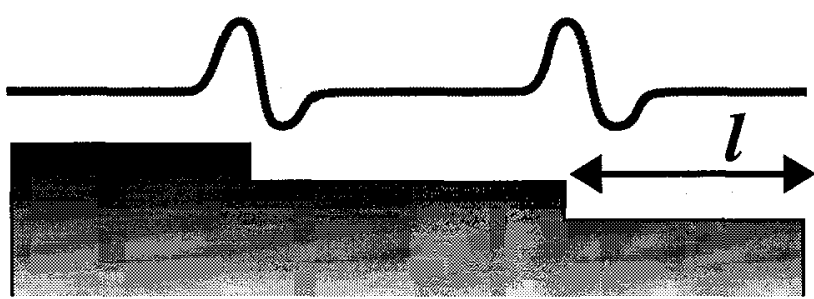

figure 6 : potentiel d'un ad-atome sur une surface vicinale trouve dans une situation très "inconfortable" (figure 6). Cet effet (effet Schwoebel) peut, par exemple, avoir un effet stabilisant (lissant) lors de la croissance sur une surface vicinale. Illustrons ceci par un modèle unidimensionnel simplifié, emprunté à Villain [6]. Soit lo la taille moyenne des terrasses, et l(x) la taille d'une terrasse particulière à la position $x$ ( $x$ est une variable macroscopique contrairement à 1 ). Le gradient local de hauteur de la surface par rapport à la pente moyenne peut s'écrire :

$$
\nabla z=\frac{1}{1}-\frac{1}{1_{0}} \approx \frac{l_{0}-1}{1_{0}^{2}}
$$

et le flux latéral d'ad-atomes capturés uniquement par la marche montante (biais total) est égal au produit du flux surfacique incident par le déplacement moyen pour atteindre la marche montante :

L'équation de conservation s'écrit alors :

$$
j=\frac{f .1}{2}
$$

$$
\frac{\partial z}{\partial t}=f-\operatorname{div}(j)=f+\frac{f \cdot l_{0}^{2}}{2} \nabla^{2} z
$$

où le signe " + " devant le deuxième terme indique le caractère stabilisant de cet effet.

Cependant, ce biais à la diffusion peut être notablement réduit si un mécanisme d'échange permet à l'adatome de descendre une marche en poussant celui qui s'y trouve (figure 5); ce mécanisme présente une barrière d'énergie plus faible que la descente directe de la marche. En dehors des marches, le mécanisme d'échange peut même constituer le processus dominant assurant la diffusion.

Les barrières d'activation de tous ces processus sont les paramètres clé de la croissance, ils font maintenant l'objet de calculs numériques utilisant des potentiels d'interaction atomiques [7] et pourront fournir les ingrédients de base pour des modèles analytiques ou pour des simulations numériques de type Monte Carlo cinétique de la croissance.

\subsection{Influence des processus hors équilibre}

Il est indispensable de tenir compte du caractère hors équilibre de la croissance de métaux en films minces pour la raison suivante : comme nous l'avons vu auparavant, l'épitaxie des métaux est dans la plupart des cas réalisée à basse température (souvent à température ambiante). Ainsi, la désorption est négligeable et le transport de matière par diffusion de surface est limité par rapport au flux incident. On est donc loin des conditions d'équilibre thermodynamique. Une première conséquence de ces conditions de croissance concerne la rugosité de la surface. En raison des fluctuations du flux, l'apport de matière n'est pas le même en différents points de la surface, ce qui conduit à une rugosité dite cinétique.

En fonction de la température, c'est à dire du rapport de la longueur de diffusion $\mathrm{x}_{S}$ à la taille moyenne des terrasses $\mathrm{L}$, on aura une cinétique de croissance différente :

-à haute température, $2 \mathrm{x}_{\mathcal{S}}>\mathrm{L}$, il y a croissance par avancée de marches

-à température moyenne, $2 \mathrm{x}_{5} \leq \mathrm{L}$, il y a nucléation bidimensionnelle sur les terrasses

-à basse température, $2 \times s<<L$, on a alors nucléation tridimensionnelle (effet Schwoebel)

Pour les métaux, c'est la deuxième situation qui a lieu à température ambiante, et on ne peut pas trop augmenter la température pour lisser la surface, sous peine d'activer l'interdiffusion en volume. Néanmoins, quand on diminue la température, on observe souvent une croissance bidimensionnelle contrairement à ce que prédit la troisième situation. Une explication possible réside dans une très petite taille d'îlots $2 D$ : l'effet Schwoebel est alors masqué parce que la plupart des sites d'ad-atomes sont près des bords de terrasses. Citons comme exemple l'homoépitaxie "platine sur platine(001)" dont la croissance est bidimensionnelle à $620 \mathrm{~K}$ et $275 \mathrm{~K}$ mais tridimensionnelle à $420 \mathrm{~K}$ [8]. Cependant, trop baisser la température entraîne une rugosité cinétique importante. Celle-ci est due au caractère aléatoire du flux 
incident d'atomes. Différents modèles de croissance conduisent à une rugosité cinétique proportionnelle à une puissance $\beta$ de l'épaisseur [6,9,10]. Expérimentalement, Chevrier [11] donne une valeur de $\beta=0,25$. Typiquement, pour un dépôt de $100 \AA$ effectué à température ambiante, on aura une rugosité de l'ordre de $10 A$. Le remède à la rugosité est d'augmenter la température, mais il faut choisir un compromis pour limiter l'interdiffusion des couches. Un autre solution consiste à augmenter l'énergie incidente des atomes : c'est le cas de la pulvérisation cathodique ou de l'ablation laser qui peuvent parfois donner de meilleurs dépôts que l'évaporation ; par exemple, la croissance de $\mathrm{Co} / \mathrm{Cu}(001)$ est meilleure en ablation laser qu'en épitaxie par jets moléculaires. Une seconde conséquence de l'écart à l'équilibre concerne les alliages. Il est possible de réaliser des alliages désordonnés alors qu'à l'équilibre des phases ordonnées peuvent exister. Il faut donc toujours garder à l'esprit que la métallurgie du matériau fabriqué peut être différente de celle exposée dans les ouvrages classiques de métallurgie.

\section{L'EPITAXIE}

\subsection{Relation d'épitaxie}

Selon la nature cristalline, l'orientation, la qualité de surface et la méthode d'élaboration utilisée, le dépôt peut être amorphe, polycristallin (texturé ou non), ou bien en relation cristallographique univoque avec le substrat. Dans ce dernier cas, on parle d'épitaxie. Il faut alors préciser la relation d'épitaxie, c'est à dire la relation d'orientation entre le substrat et le dépôt. L'information nécessaire réside dans la donnée des plans cristallins du substrat et du dépôt parallèles à l'interface et de deux directions paralièles du substrat et du dépôt. Par exemple, Au(110)[001] // $\mathrm{Fe}(211)$ [011] signifie que la direction de

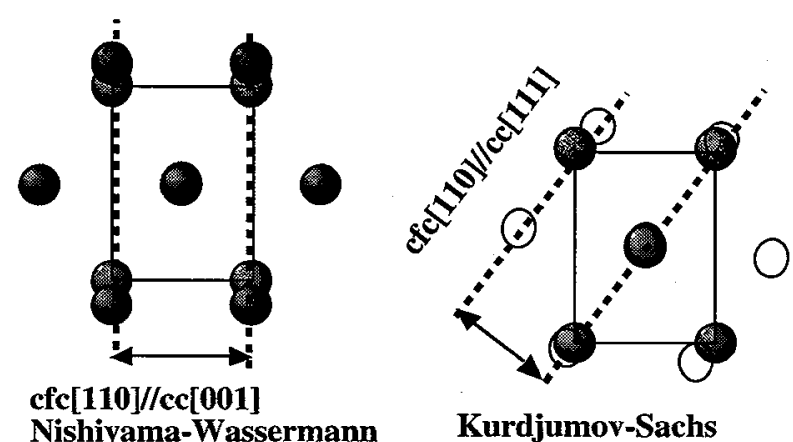

Nishiyama-Wassermann

figure 7 : Relations d'épitaxie entre CFC (111) et CC (110) croissance de l'or sur la surface (211) du fer est (110) et que dans le plan les directions [011] du fer et [001] de l'or sont parallèles.

Des critères qui utilisent des considérations géométriques simples ou énergétiques ont été proposés pour rendre compte et tenter de prévoir les relations interfaciales [12,13,14], par exemple le critère de ligne invariante [15] ou la théorie du réseau Zéro [16]. Les relations d'épitaxie entre un métal cubique à faces centrées et un métal cubique à corps centré selon leurs faces denses (111) et (110) respectivement, relèvent du critère de la ligne invariante (figure 7). Ce critère dit que les deux cristaux s'orientent de façon qu'il y ait une direction dans le plan de l'interface qui ne subisse pas de déformation.

\subsection{Mécanismes de relaxation}

Lorsqu'il n'y a pas de relation d'épitaxie possible entre les phases naturelles du dépôt et du substrat, ( par exemple à cause d'une forte différence de paramètre de maille), il arrive qu'une phase nouvelle soit stabilisée par le substrat [17] ; par exemple, la phase cubique à faces centrées du Co est stabilisée sur le $\mathrm{Cu}(001)$, elle devient cubique centrée sur le Fe(001) [18]. Si le désaccord des paramètres de maille est plus faible, le dépôt s'accorde au substrat de manière pseudomorphe par déformation élastique. Dans les deux cas, l'énergie élastique et/ou l'énergie de la phase métastable augmentent proportionnellement avec l'épaisseur du dépôt. Par conséquent, le dépôt finit par relaxer vers sa phase naturelle pour une épaisseur critique qui dépend du mécanisme de relaxation : changement de structure similaire à une transformation martensitique, instabilités de la surface du dépôt [19], apparition de discommensurations ou de dislocations [20], croissance $3 \mathrm{D}$, etc....

\subsection{1 instabilité de la surface}

Lorsque le dépôt est cohérent, (continuité des plans atomiques du substrat dans la couche déposée) l'énergie élastique due au désaccord de paramètre de maille est proportionnelle à l'épaisseur. On peut montrer que l'énergie élastique pour une couche dont la hauteur varie sinusoïdalement (figure $8 b$ ) est inférieure à celle 
d'une couche de hauteur homogène de même volume (figure 8a). Cependant, cette réduction de l'énergie se paie par un supplément d'énergie superficielle. L'ondulation de la surface requiert un transport de matière qui peut se faire par la diffusion de surface, celle-ci étant déjà activée à la température de dépôt pour les métaux. Cette instabilité donne lieu à une ondulation de la surface dont la longueur d'onde caractéristique résulte d'un compromis entre l'énergie élastique qui constitue la force motrice, l'énergie de surface qui favorise les grandes longueurs d'onde et la diffusion en surface qui tend au contraire à favoriser les petites longueurs d'onde. On peut modéliser cet effet à partir du champ de potentiel chimique des ad-atomes soumis à la contrainte tangentielle et à la tension de surface due à la courbure locale :

$$
\mu=\mu_{0}-\gamma \Omega \frac{\partial^{2} \mathrm{~h}}{\partial \mathrm{x}^{2}}+\frac{\Omega}{2 \mathrm{M}}\left\{\sigma_{\mathrm{tt}}(\mathrm{x})^{2}-\sigma^{2}\right\}
$$

L'équation d'évolution de la hauteur s'écrit alors :

$$
\frac{\partial \mathrm{h}}{\partial \mathrm{t}}=\frac{\mathrm{D} \Omega \delta}{\mathrm{kT}} \frac{\partial^{2} \mathrm{~h}}{\partial \mathrm{x}^{2}}
$$

A l'instar de la théorie de la décomposition spinodale, les premiers stades de l'instabilité donnent une ondulation de la surface. On retient la longueur d'onde dont l'amplitude s'accroît le plus rapidement. Ce type d'instabilités proposées théoriquement n'a cependant pas été clairement mis en évidence expérimentalement et constitue un sujet encore très controversé.

\subsubsection{Introduction de dislocations}

Un autre mode de relaxation largement rencontré consiste dans l'introduction de dislocations (figure $8 \mathrm{c}$ ) qui glissent jusqu'à l'interface entre la couche contrainte et le substrat ou au voisinage de l'interface (dislocations en "stand off') quand les modules élastiques de part et d'autre de l'interface sont différents. En suivant l'approche de Matthews [20], on peut exprimer l'énergie élastique totale comme la somme d'un terme d'énergie élastique de déformation homogène proportionnel à l'épaisseur $h$ et d'un terme d'énergie
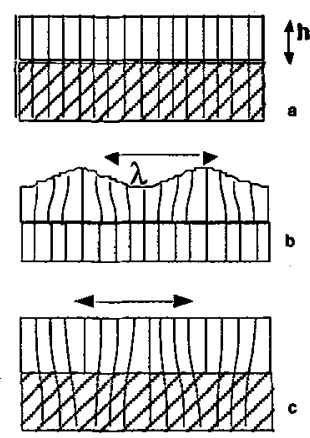

figure 8 : mécanisme de relaxation.

a) couche contrainte

b) instabilité de surface

c) introduction de dislocations

élastique dû au champ de déformation autour des dislocations proportionnel seulement au logarithme de l'épaisseur :

$E=\frac{2 G(1+v)}{1-v} h \varepsilon^{2}+(f-\varepsilon) \frac{G b}{2 \pi(1-v)}\left\{\log \left(\frac{h}{b}\right)+1\right\}$ avec $f=\frac{a_{\text {substrat }}}{a_{\text {dépôt }}}-1$,

$b$ est le module du vecteur de Burgers, $v$ le coefficient de Poisson et $G$ le module de cisaillement.

Un raisonnement basé sur une minimisation de l'énergie totale montre qu'au-delà d'une certaine épaisseur "critique" $h_{\mathcal{C}}$, l'introduction de dislocations à l'interface est plus avantageuse. Depuis les travaux de Matthews, des modèles plus poussés ont été proposés, tenant compte du système de glissement réellement actif, de l'anisotropie élastique, de la différence de constantes élastiques entre le substrat et le dépôt etc.... L'épaisseur pour laquelle commence la relaxation peut être plus grande que ne l'indiquent les calculs d'équilibre (minimisation de l'énergie élastique); on met alors en cause une limitation cinétique de la nucléation et de la propagation des dislocations.

\subsection{Influence de l'empilement}

Lors de la réalisation de multicouches A/B pour étudier des propriétés physiques diverses ou pour les applications (optiques $\mathrm{X}$, enregistrement magnétique), on cherche en général à minimiser la rugosité des interfaces et donc à obtenir une croissance bidimensionnelle pour toute la couche. Les modèles d'équilibre basés sur l'énergie superficielle interdisent la croissance bidimensionnelle simultanée des couches A/B et $\mathrm{B} / \mathrm{A}$. Néanmoins, de nombreux exemples montrent que la réalisation de super réseaux plans est heureusement possible. Citons les cas de $\mathrm{Fe} / \mathrm{Cu}[21] \mathrm{Fe} / \mathrm{Cr}$ [22] et $\mathrm{Fe} / \mathrm{Ir}$ [23].

\section{CONCLUSION}

Les mécanismes de croissance cristalline sont donc nombreux, complexes et souvent imbriqués, ce qui rend difficile la compréhension des phénomènes observés. Néanmoins, l'étude de la croissance des métaux a bénéficié sans aucun doute du savoir-faire obtenu sur la croissance des semi-conducteurs, étudiés bien avant celle des métaux. On a pu constater ainsi que les mécanismes de base sont très souvent les 
mêmes, mais que les phénomènes collectifs (mode de croissance, relaxation) peuvent être très différents principalement parce que les métaux ne sont pas des matériaux covalents comme les semi-conducteurs.

Il faut également mentionner que les mécanismes décrits ont essentiellement été observés à l'aide d'étude de croissance sous ultra-vide. En outre, d'autres phénomènes physiques ou/et chimiques interviennent pendant la croissance en pulvérisation cathodique ou en ablation laser par exemple. On connaît encore mal l'influence de l'énergie des ions sur les mécanismes de croissance en pulvérisation cathodique ou bien des phénomènes de "trempe" des atomes, molécules ou agrégats arrivant à la surface du substrat en ablation laser. La croissance et plus particulièrement l'épitaxie fait et fera donc encore l'objet de nombreuses études dans l'avenir.

\section{Remerciement.}

Les auteurs remercient S. Bouat et G. Abadias (DRFMC Grenoble).

\section{Références}

[1] Baibich M.N.et al, Phys. Rev. Lett., 61, (1988), 2472

[2] Bruno P. et Chappert C., Phys. Rev. Lett., 67, (1991), 1602 et 2592(E)

[3] Chatillon C. et Massies J., Metallic Multilayers, 59,60 (1990) 229.

[4] Masson A. Metallic Multilayers, 59,60 (1990) 41.

[5] Bermond J. M.. Metallic Multilayers, 59,60 (1990) 1.

[6] Villain J. J. Phys. I1 (1991) 19.

[7] Deutsch T., Thèse INPGrenoble, (1995) et T. Deusch, P. Bayle, F. Lançon and J. Thibauit, J. Phys. : Condens. Matter 7 (1995) 6407.

[8] Kunkel R.,Poelsema B. Verheij L.K.and Comas G., Phys. Rev. Let., 65 (1990) 733.

[9] Kardar M., Parisi G. Zhang Y.C. ,Phys. Rev. Let., 56 (1986) 889.

[10] Lai Z.-W.et Das Sarma S., Phys. Rev. Lett. 66 (1991) 2348

[11] Chevrier J. et al, Euro Lett. 16 (1991) 737.

[12] Sutton A.P. and Balluffi R. W.,Acta Metall. 35 (1987) 2177.

[13] Van-Der-Merve J. H., Phil. Mag A 45 (1982) 127. et

[14] Riess H. J. of Appl. Phys. 39 (1968) 5045.

[15] Dahmen U. Acta Metall. 30 (1982) 63.

[16] Bollmann W., Surf. Sci. 31 (1972) 1.

[17] Brunisma R.and Zangwill A. J. Phys 47 (1986) 2055.

[18] Li H. and Tonner B. P., Phys. Rev. B 40 (1989) 10241.

[19] Srolovitz D. J., Acta Met. 37 (1989) 621.

[20] Matthews J. W., Epitaxial Growth, B (J.W. Matthews, New York, 1975) 563

[21] Heinrich B. et al, Phys. Rev. Lett. 64 (1990) 673.

[22] Purcell S.T. et al, Phys. Rev. Lett. 67 (1991) 903.

[23] Andrieu S.et al, J. Appl. Phys. 77 (1995) 1308. 